\section{Die Testphase}

Insgesamt 500 Praxen, davon 125 Zahnarztpraxen, und sechs Krankenhäuser erproben seit Mitte November letzten Jahres in Schleswig-Holstein Nordrhein-Westfalen und Rheinland-Pfalz, inwieweit der Online-Abgleich der Versichertenstammdaten über die Telematikinfrastruktur (TI) unter realen Bedingungen und mit Echtdaten praxistauglich ist. Bis Ende April 2017 wurden in der Testregion Nordwest bereits 500.000 Onlineprüfungen durchgeführt und somit das quantitative Erprobungsziel erreicht. Basierend darauf haben die Gematik-Gesellschafter Anfang Juni 2017 die Freigabe für den Online-Produktivbetrieb erteilt.

Die Anbindung an das digitale Netz des deutschen Gesundheitswesens erfordert ein neues Bauteil, den Konnektor. Er verbindet in einem virtuellen privaten Netzwerk die Praxis- bzw. Krankenhaus-IT-Systeme mit der TI und macht die TI-Anwendungen nutzbar. Über diese werden Daten laut einer Pressesprecherin der Gematik ausschließlich transportiert, nicht gespeichert. Außerdem steuert der Konnektor den Zugriff auf die im Netzwerk der Praxis angeschlossenen Kartenterminals und die darin gesteckten ChipKarten.

Als erste Anwendung ist die Online-Aktualisierung der elektronischen Gesundheitskarte am Konnektor verfügbar. Bei dieser Anwendung werden die Adressdaten und der Versicherungsstatus mit der Krankenkasse abge- glichen. Dabei werden weder Gesundheitsdaten übertragen, noch kann die Krankenkasse ermitteln, bei welchem (Zahn-)Arzt der Versicherte seine Karte gesteckt hat. Daneben wird der Konnektor noch andere Aufgaben erfüllen, beispielsweise das Verschlüsseln und Signieren von medizinischen Dokumenten. Laut Presseabteilung der Gematik besitzt der Konnektor "ein sehr hohes Sicherheitsniveau“: Die Konnektoren werden vom Bundesamt für Sicherheit in der Informationstechnik zertifiziert, bevor sie die Zulassung der gematik erhalten.

Ein Konnektor kann nur dann eine Verbindung zur Telematikinfrastruktur aufbauen, wenn sich die jeweilige (Zahn-)Arztpraxis, Psychotherapeutenpraxis, das jeweilige MVZ, das Krankenhaus oder die Apotheke ausweisen. Dazu dient eine Praxis- beziehungsweise Institutionskarte, im ITDeutsch "SMC-B“ genannt. Am ehesten vergleichbar ist sie mit den SIM-Karten von Mobiltelefonen. Die SMC-B steckt in einem Kartenterminal und wird in der Regel morgens durch PIN-Eingabe vom Praxispersonal aktiviert. Die SMC-B-Karte bestätigt dem zentralen Netzwerk, dass der Zugriff über die Praxis $X$, das MVZ Y oder das Krankenhaus Z erfolgt. Neben ihrer Funktion als "Praxisausweis" hat sie auch wesentliche Sicherheitsfunktionen. Sie kann zum Beispiel zum Austausch von verschlüsselten elektronischen Nachrichten verwendet werden.

mar gehe ich davon aus, dass die Datensicherheit gegeben ist. Risiken gibt es immer. Die gibt es jedoch auch in der analogen Welt. Wichtig ist es, dass sich die Kolleginnen und Kollegen mit diesen Risiken, aber auch mit den Chancen der Telematikinfrastruktur sachlich auseinandersetzen. Genauso wichtig ist es, dass sie bei der Online-Anbindung ihrer Praxis nur auf zertifizierte Dienstleister und zugelassene Produkte, die die Sicherheitsvorgaben erfüllen, setzen.

DFZ: Der Konnektor ist entscheidend für die TI-Anbindung. In welcher Hinsicht testen Sie das Gerät in Ihrer Praxis?

Koller: Unsere Aufgabe als Testpraxis ist es, das gesamte System auf Praxistauglichkeit und Funktionalität im Echtzeitbetrieb einer Zahnarztpraxis zu erproben. Wie lässt sich der Konnektor anschließen? Fügt er sich problemlos in die Praxis-IT ein? Ist die Telematikinfrastruktur stabil und sicher? Funktioniert der Datenabgleich? Und kommen die Praxismitarbeiter zurecht?

Es wäre verwunderlich gewesen, wenn der Konnektor unseren Praxisalltag anfangs nicht beeinflusst hätte. Zunächst einmal lief die Installation holprig. Startschwierigkeiten bestanden auch darin, dass der Konnektor häufiger keine Onlineverbindung herstellen konnte oder er einfach abstürzte. Zudem hakte es, da das System aus Konnektor, Kartenterminal und Praxisverwaltungssystem noch nicht vollständig kompatibel war. Solche Probleme tauchen jetzt nach Beendigung der Testphase kaum noch auf - und wenn doch, hilft die Hotline des Herstellers unkompliziert und schnell weiter. Am Ende der Testphase können wir sagen, dass das System stabil läuft.

Der Nutzen des Versichertenstammdatenmanagements liegt darin, dass wir neue oder geänderte Patientendaten direkt in unser Praxisverwaltungssystem per Mausklick übernehmen können, statt sie per Hand erfassen zu müssen. Das wiegt den kleinen zeitlichen Mehraufwand beim Einlesen der Daten auf.

DFZ: Was könnte noch besser laufen?

Koller: In der Testphase hatten wir 17 ungültige elektronische Gesundheitskarten, die wir nicht einlesen konnten. Der Grund dafür war meist ein einfacher: Die Patienten hatten zwar eine online-fähige Versicherungskarte von ihrer Krankenkasse bekommen, diese aber zu Hause vergessen. Die Kassen müssen ihre Versicherten zukünftig deutlicher darauf hinweisen, ausschließlich die neue Gesundheitskarte zu nutzen - auch wenn die alte Karte noch nicht abgelaufen ist. Seit November hatten wir nur einen Patienten, der tatsächlich nicht versichert war. Ihn haben wir an die Krankenkassen verwiesen mit der Bitte, seinen Versicherungsstatus zu klären.

Wir müssen uns gegenüber dem Kartenlesegerät jeden Morgen mit einer PIN ausweisen. Das ist aus Sicherheitsgründen auch gut und richtig. Entweder denkt die Mitarbeiterin morgens von sich aus an die Eingabe oder sie wird beim ersten Einlesen einer Karte daran erinnert. Es wäre hilfreich, wenn das Kartenterminal automatisch nach der PIN fragen würde.

DFZ: Auf welche Weise und wie häufig wird Ihr Feedback dazu erfragt? Koller: Die gematik hat den Lehrstuhl für Gesundheitsmanagement der Friedrich-Alexander-Universität Erlangen-Nürnberg beauftragt, die Testphase wissenschaftlich zu evaluieren. In der Erprobungsphase wurden wir insgesamt fünfmal online befragt. Wir wurden dabei gebeten, zum Beispiel Feedback zu geben zur Installation des Konnektors und des eHealth-Kartenterminals oder zum laufenden Betrieb des Versichertenstammdatenmanagements. Die Befragungen werden ausgewertet und deren Ergebnisse in einem Abschlussbericht der gematik zur Verfügung gestellt.

DFZ: Behalten Sie das Gerät nach Ablauf der Testphase in Ihrer Praxis? Koller: Stand der Dinge ist, dass die Testteilnehmer die technischen Komponenten aus der Erprobungsphase auch im Produktivbetrieb behalten werden - zumindest bis auf Weiteres. Denn in der zweiten Phase der Erprobung müssen beide Komponenten ausgetauscht werden. Für die Tests der qualifizierten elektronischen Signatur (QES) und der sicheren Kommunikation der Leistungserbringer untereinander (KOM-LE) sind weder der derzeitige Testkonnektor noch das Kartenterminal technisch ausgerüstet bzw. zertifiziert.

Interview: Maike Raack 